
Disease: A Population-Based Study in South Korea

\title{
Youngju An
}

Baekseok Culture University

Hyojin Kim ( $\square$ opt9580@gmail.com )

Baekseok University

\section{Research Article}

Keywords: Sleep Disorders, Mental Health, Dry Eye Disease, Population-Based Study, South Korea, severe discomfort, mild ocular irritation, fatigue, pain, visual disturbance, foreign body sensation

Posted Date: August 17th, 2021

DOl: https://doi.org/10.21203/rs.3.rs-799158/v1

License: (c) (1) This work is licensed under a Creative Commons Attribution 4.0 International License. Read Full License 


\section{Abstract}

Dry eye disease (DED) is a multifactorial disease of the ocular surface that causes severe discomfort, mild ocular irritation, fatigue, pain, visual disturbance, and a foreign body sensation. Stress, depression, and sleep disorders are considered risk factors for DED. Our aim was to investigate the association between mental health, sleep disorders, and DED in Korean adults. This population-based, cross-sectional study examined ophthalmologic data of 16,471 Koreans aged $\geq 20$ years from the 2010-2012 Korea National Health and Nutrition Examination Survey (KNHANES). DED was based on a medical diagnosis by a doctor, and data on mental health and sleep disorders were obtained using questionnaires. Multiple logistic regression analysis was conducted to examine the association between mental health, sleep disorders, and DED, and we also adjusted for possible covariates. Short sleep duration ( $\leq 5 \mathrm{~h} / \mathrm{night}$ ), stress perception (yes), and depressive symptoms (yes) were significantly associated with the risk of DED even after correcting for demographic factors, lifestyle factors, and medical factors. Combined short sleep duration, stress, and depression were most strongly associated with DED, and thus ophthalmologists must be aware of the possibility of a higher prevalence of sleep disorders, stress, and depression in patients with DED.

\section{Introduction}

Dry eye disease (DED) is a growing public health problem within ophthalmology ${ }^{1}$. It is defined as a multifactorial disease of the tear ducts and ocular surface, which is accompanied by increased osmolality of the tear film and inflammation of the ocular surface ${ }^{2}$. The prevalence of dry eye disease among middle-aged and elderly persons ranges from $7-34 \%^{3}$. The incidence of DED, which has always been one of the most common ophthalmic diseases, has been markedly increasing in industrialized countries ${ }^{4}$.

DED results in severe discomfort, mild ocular irritation, fatigue, pain, visual disturbance, a foreign body sensation, and tear film instability, which can potentially damage the ocular surface ${ }^{2,5,6}$. It interferes with activities of daily living ${ }^{7}$, thus negatively affecting patients' mental health as well as productivity at work ${ }^{8,9}$. DED is recognized as a major public health problem worldwide ${ }^{6}$, and it can place a significant financial burden on the healthcare system ${ }^{10,11}$.

Risk factors for DED have been reported in several studies. These risk factors include old age, female sex, smoking status, wearing contact lenses, systemic medications, video display use, and a history of ocular surgery $3,7,12-15$. Depression, sleep disorders, and stress are also considered to be risk factors for DED based on recent reports $9,16,17$. There has recently been increased medical interest in the psychiatric pathophysiology of patients with $\mathrm{DED}^{18}$. According to a previous study, respondents with shorter sleep durations had more DED symptoms than those with normal sleep durations ${ }^{16}$. However, this report has not controlled for history of ocular surgery, including refractive and cataract surgery, although these are 
common procedures in ophthalmic clinics. DED is one of the most common complications after LASIK and cataract surgery ${ }^{19,20}$.

Therefore, this study aimed to determine the relationship between DED and psychiatric factors of a representative sample of Korean adults aged $\geq 20$ years in a population-based study using the 20102012 Korea National Health and Nutrition Examination Survey (KNHANES) data. In addition, we attempted to ascertain whether those associations would be affected by a comorbidity of psychiatric factors, including short sleep durations, stress perception, and depressive symptoms after adjusting for age, sex, lifestyle, and medical factors.

\section{Patients And Methods}

\section{- Study design and population}

The Korean National Health and Nutrition Examination Survey (KNHANES) is a nationwide populationbased cross-sectional health examination and survey conducted regularly by the Korea Centers for Disease Control and Prevention under the Ministry of Health and Welfare. The fifth KNHANES was conducted from 2010 to 2012. A stratified, multistage probability sampling design is used for the selection of household units that participate in the survey, which ensures that each year's survey results represent the entire general population of South Korea. In total, 31,596 people participated in fifth KNHANES V, representing 11,400 households (3,800 households/year) across 576 national districts (192 national districts/year). The response rates for the 2010, 2011, and 2012 KNHANES were $81.9 \%$ $(n=8,958), 80.4 \%(n=8,518)$ and $80.0 \%(n=8,058)$, respectively (Figure 1$)$.

Participants in the survey undergo a health interview, comprising health examinations, an ophthalmic interview and ophthalmic examinations, and a nutrition survey. A health interview and examinations were performed by trained interviewers and medical staff at the mobile examination center. The ophthalmic survey was verified by the Epidemiology Survey Committee of the Korean Ophthalmological Society and the detailed di-agnostic criteria for eye conditions used in this study are described elsewhere ${ }^{12}$.

This study followed the tenets of the Declaration of Helsinki for biomedical research and was approved by the Institutional Review Board (IRB) of Korea Center for Disease Control and Prevention (KCDC) (No: 2010-02CON-21-C, 2011-02CON-06-C, 2012-01EXP-01-2C). Written informed consent was obtained from all participants when the 2010 2012 KNHANES were conducted.

\section{- Participants and data selection}

We included 25,534 participants who had completed ophthalmological examinations in the KNHANES 2010-2012. We excluded participants aged $<20$ years $(n=6,140)$, or those who had no DED data $(n=2,599)$. We further excluded those without mental health data $(n=324)$. Finally, after implementing these exclusion criteria, 16,471 participants were included for analysis in the present study (Figure 1). 
DED was defined as dry eye diagnosed by a physician, who referred to a dry eye questionnaire used in previous studies ${ }^{12,9}$. A questionnaire related to DED was distributed, which includes the following question: "Have you ever been diagnosed with DED by a physician?" (Participants were asked the aforementioned question with an emphasis on "by a physician."), and this question could be answered as "yes" or "no." Sleep duration was assessed via the self-administered question, "How many hours do you usually sleep a day?" The third edition of the International Classification of Sleep Disorders generally classifies long sleepers as those sleeping for $\geq 9-10 \mathrm{~h}$, and short sleepers as those sleeping for $\leq 5-6$ $h^{36,37}$.

Therefore, this study classified sleep time as $\leq 5,6,7,8$, or $\geq 9 \mathrm{~h}$ /day according to the intervals used in the previous study ${ }^{38}$. Participants were asked to respond "yes" or "no" to the following mental health questions: "Have you ever had a lot of stress?" and "Have you ever felt depressed for 2 continuous weeks?" We defined the participants as having a severe mental health problem when they answered positively regarding shorter sleep duration, a lot of stress perception, and depression symptomology.

Questionnaires were used to collect data on age, sex, education, household income, occupation, smoking, regular exercise, daily sun exposure, pregnancy, and history of ocular surgery. Subjects were classified into six age groups: $20-29,30-39,40-49,50-59,60-69$, and $\geq 70$ years. Educational attainment was classified into the following categories: less than an elementary school education, middle school, high school, and more than university. Household income was collapsed into quartiles. Occupation was classified as blue collar (agriculture workers, forestry workers, fishery workers, craft and related trade workers, plant and machine operators and assemblers, and simple labor), white collar (managers, professionals, clerks, and service or sales workers), and those who were un-employed for any reason. Current smoking was classified as yes or no (current nonsmokers were either never smokers or past smokers). Regular exercise was defined as walking $>30$ minute least 5 days a week. Daily sun exposure was classified into categories of $<2 \mathrm{~h}, 2 \sim 5 \mathrm{~h}$, and $\geq 5 \mathrm{~h}$ of typical exposure per day. Pregnancy was assessed via the question, "Are you currently pregnant?" History of previous ocular surgery was evaluated via the question, "Have you ever had ocular surgery in the past?"

Anthropometric measurements were used to collect data on body mass index (BMI), diabetes, hypertension, thyroid disease, and rheumatoid arthritis. BMI was defined as underweight BMI $(<18.5$ $\mathrm{kg} / \mathrm{m} 2)$, normal BMI ( $\leq 18.5$ to $<25 \mathrm{~kg} / \mathrm{m} 2)$ and overweight/obesity $(\geq 25 \mathrm{~kg} / \mathrm{m} 2)^{38}$. Diabetes was evaluated through the question: "Have you ever diagnosed with diabetes by a physician?" Hypertension was defined as systolic blood pressure $>160 \mathrm{mmHg}$ and/or diastolic blood pressure $>90 \mathrm{mmHg}$, measured at the medical examination, or currently taking antihypertensive medications ${ }^{38}$. Thyroid disease and rheumatoid arthritis diagnoses were classified into combined yes or no categories through the question: "Have you ever been diagnosed with thyroid disease or rheumatoid arthritis by a physician?"

- Statistical analyses 
Weighted analysis was used to more accurately reflect the true population statistics. The data are expressed as unweighted numbers and weighted proportions (\%). The chi-square test was used to compare the demographic, lifestyle, and medical characteristics of the study population between participants with DED and those without DED. Multiple logistic regression analysis was conducted to examine the odds ratios (ORs) and 95\% confidence intervals (Cls) for the association between mental health related characteristics (sleep duration, stress perception, depressive symptoms, and severe mental health) and DED. Demographic factors (age and sex), lifestyle factors (education, occupation, BMI, smoking, regular exercise, and daily sun exposure) and medical factors (pregnancy, thyroid disease, and history of ocular surgery) were used as covariates for calculating adjusted odds ratios (aORs). Statistical analyses were performed using SPSS 18.0 version (SPSS Inc., Chicago, IL, USA). All reported $P$ values are two-tailed, and values $<0.05$ was considered the threshold for statistical significance.

\section{Results}

Among the 25,534 people who participated in KNHANES V (2010-2012), there were 19,394 participants who were aged $\geq 20$ years, of whom 2,599 participants did not have data on DED diagnosis and 324 participants did not have data on mental health. Those participants were excluded, leaving a total of 16,471 participants selected as final participants (Figure 1).

In adults aged $\geq 20$ years, the estimated DED prevalence (standard error [SE]) was $16.19 \%(0.56)$, with a prevalence of $10.61 \%$ (0.57) among males and $21.55 \%$ (0.75) among females. The association between sleep duration and weighted DED prevalence is presented in Figure 2. The weighted DED prevalences (SE) were $19.40 \%$ (1.04), $16.68 \%(0.82), 15.22 \%(0.77), 15.09 \%$ (0.81), and $15.66 \%$ (1.41) for participants with sleep durations of $\leq 5,6,7,8$, and $\geq 9 \mathrm{~h} /$ night, respectively.

The association between stress perception and weighted DED prevalence is presented in Figure 3. Weighted DED prevalence (SE) was $20.05 \%$ (0.92) among those with higher stress perception and $14.72 \%$ (0.57) among those with no/low stress perception. The association between depressive symptoms and weighted DED prevalence is presented in Figure 4. The association between severe mental health [combined short sleep duration $(\leq 5)$, stress, and depression] and weighted DED prevalence is presented in Figure 5.

Demographic, lifestyle, and medical characteristics of participants in the present study are shown in Table 1. Regarding demographic characteristics, we found that compared with those without DED, there were many cases with DED among participants aged $<20$ years or those aged $>50$ years, among women, among those whose education level was less than an elementary school educational attainment, and among college students, unemployed participants, and white collar workers. Regarding lifestyle characteristics, there were more cases with DED among those with normal or underweight BMI, current nonsmokers, those not exercising regularly, and participants with daily sun exposure of $<2 \mathrm{~h}$ per day compared with those without DED. Regarding clinical characteristics, there were more cases of DED among pregnant participants, those with thyroid disease, and those with previous ocular surgery. 
Mental health related characteristics of subjects with and without DED are shown in Table 2. Compared with participants without DED, those with DED frequently reported a sleep duration of $\leq 5 \mathrm{~h} / \mathrm{night}$ or $\leq 6$ $\mathrm{h} / \mathrm{night}$ in many cases, as well as a greater frequency of greater stress perception, depressive symptomology, and severe mental health symptomology.

Table 3 shows the results of multiple logistic regression analyses presenting the association of DED with related mental health characteristics. Even after adjusting for demographic factors, lifestyle factors, and medical factors, people who sleep for $<5 \mathrm{~h}$ a night ( $\mathrm{OR}=1.42,95 \% \mathrm{Cl}=1.06-1.90)$, those with greater stress perception ( $\mathrm{OR}=1.71,95 \% \mathrm{Cl}=1.37-2.14)$, those reporting depressive symptomology (OR=1.37, 95\% $\mathrm{Cl}=1.06-1.77)$, and those with severe current mental health presentation $(\mathrm{OR}=1.91,95 \% \mathrm{Cl}=1.07-3.39)$, had a higher risk of developing DED.

\section{Discussion}

Upon exploring the correlation between DED and psychiatric factors among Koreans aged $\geq 20$ years, this study showed that the risk of DED increased with short sleep duration, stress perception, and depressive symptoms. This result was in keeping with the results of previous studies. This study also showed that the risk of DED was the highest in those with severe mental health issues (those experiencing all three aforementioned factors).

DED is a common irritating eye disease that causes pain, discomfort, burning, a foreign body sensation, and blurred vision. Previous studies have evaluated the association between psychological problems and eye disease, such as age-related macular degeneration (AMD), cataract, glaucoma, and DED ${ }^{21}$. DED has a known association with mental health problems, such as depression and anxiety ${ }^{22}$. A meta-analysis evaluating the correlation between eye disease and depression showed that the prevalence of depression was $29 \%$ among patients with DED, $25 \%$ in patients with glaucoma, $24 \%$ in patients with AMD, and $23 \%$ in patients with cataract, showing the highest prevalence among patients with $\mathrm{DED}^{21}$.

Wan et al. ${ }^{22}$ stated that the depression and anxiety score in patients with dry eye symptoms were higher than that in the control group, which was similar to the results obtained from this study. Ayaki et al. ${ }^{23}$ evaluated the correlation between sleep, anxiety, and depression in 301 patients with DED and 202 patients with other ocular surface diseases (chronic conjunctivitis/ allergic conjunctivitis). They showed that the mild to severe DED group showed a higher proportion of patients presenting with problems with sleep quality based on a Pittsburgh Sleep Quality Index of $\geq 6$, as well as depression symptomology based on a Hospital Anxiety and Depression Scale Depression Subscale score of $\geq 5$, when compared with the chronic conjunctivitis or allergic conjunctivitis groups.

Lee et al. ${ }^{16}$ evaluated the association between sleep duration and dry eye syndrome (DES) in Korean adults. They showed that the prevalence of DES was 1.20 and 1.29-fold higher in the short sleep duration group ( $5 \mathrm{~h} /$ day) and in the severe sleep disruption group ( $\leq 4 \mathrm{~h} /$ day), respectively, compared with the optimal sleep group ( $6 \sim 8 \mathrm{~h} /$ day), showing that the prevalence of DES increased with shorter sleep 
duration. This is thought to be due to the stress from sensory discomfort or optical disturbance experienced by patients with DED as well as sleep difficulty from inflammatory processes (pain, brightness due to in-complete eye closure, and others) during sleep. Previous studies also show that persistent positive airway pressure, a treatment option for obstructive sleep apnea, may exacerbate DED $^{24}$.

Depression is more closely associated with dry eye symptoms compared with dry eye signs ${ }^{25,26}$. Irritating ocular surface symptoms exert negative effect on visual performance and daily activity ${ }^{27}$. Momoko et al. ${ }^{28}$ evaluated the association between psychiatric symptoms and objective measures (BUT, Schirmer value, Fluorescein staining score) in 56 patients with DED. To quantify psychiatric symptoms, the Montgomery-Asberg Depression Scale (MADRS) and HAM-A (Japanese version of the Hamilton Rating Scale for Anxiety) scales were used. Subjects were divided into those with normal MADRS and HAM-A scores and those with high MADRS and HAM-A scores to compare the differences in subjective symptoms of DED with objective measures. There was a significant difference in subjective symptoms of DED, whereas no significant difference was observed in the objective measures (BUT, Schirmer value, fluorescein staining score).

Galor et al. ${ }^{29}$ stated that patients with depression and anxiety can have central sensitization, which may affect pain perception. In other words, patients with depression or anxiety may react more sensitively to ocular sensation compared to control participants ${ }^{22}$ and experience persistent irritation from eye dryness, and this in turn can cause or exacerbate mood disorders ${ }^{30}$.

Furthermore, inflammatory cytokines play an important role in the pathogenesis of depression by triggering unhealthy behavior. Lymphocytes of a patient with depression activate the production of IL-1b, IL-6 and TNF- $a$ and such inflammatory pathways are known to cause neuronal damage through oxidative and nitrosative stress ${ }^{31,32}$. Mrugacz et al. ${ }^{33}$ explored the correlation between inflammatory cytokine levels in tears and depression among 32 (14 male, 18 female) DED patients with an average age of 44.21 years, observing that patients with depression have significantly higher inflammatory cytokines (IL-6, IL-17, and TNF- a) levels in tears than control participants, which reflects the severity of local immunological changes. In particular, locally increased IL-17 and TNF-a levels in tear fluid can be considered a result of DED progression, which implies that IL-17 and TNF-a levels can be used as predictive factors for clinical inflammation in dry eye patients with depression ${ }^{33}$.

In some patients with DED, tear production may have been compromised from using somnifacients containing anticholinergic agents ${ }^{34}$. Human conjunctival epithelium contains muscarinic and adrenergic receptors, which implies that antidepressants could plausibly affect the ocular surface ${ }^{38}$. Chronic exposure to histamines and 5-hydroxytryptamine changes function through secretory processes, and neuronal release of 5-hydroxytryptamine could plausibly affect acute control of lacrimal secretion. Zheng et al. ${ }^{21}$ stated that chronic painful symptoms from dry eye disease can induce depression and that medications used to treat depression can cause or exacerbate dry eye disease. Ayaki et al. ${ }^{23}$ stated that 
steroid or mucin secretagogue eye drops (diquafosol and re-bamipide) used to relieve severe DED symptoms can cause distress. Sleep deprivation and medication history due to psychiatric disorders may partially inhibit tear secretion, since lacrimal secretion is under neural regulation.

Thus far, not much is known regarding the pathogenesis of DED, and it is yet unclear whether depression exacerbates DED. Ayaki et al. ${ }^{18}$ hypothesized that DED causes distress, which can in turn give rise to depression and sleep disorders. Furthermore, eye pain and exposure to light from DED can cause sleep disturbance, which would in turn lead to sleep disorders. The inverse may also be possible, such that the presence of sleep disorder can cause DED and depression.

Irritating ocular symptoms can exert negative influence on visual performance ${ }^{23}$ through tear instability from DED that leads to blurred vision, which may hinder daily activities. Furthermore, DED patients can experience stress from sensory discomfort or optical disturbance, and the condition itself can cause psychological distress. DED patients experience pain and brightness due to incomplete eye closure, which can cause sleep disturbance. Such disturbance can produce inflammatory processes. Patients with Sjogren's syndrome are known to experience various sleep disorders such as pain, night awakenings, long sleep latency, and obstructive sleep apnea ${ }^{18}$.

A major strength of this study is the fact that it is the first large-scale population-based study that corrects for history of ocular surgery, including refractive and cataract surgery, and evaluates the association between DED and mental health and sleep disorders. Previous studies among Asians ${ }^{9,16}$ did not consider history of ocular surgery, although DED is the most common complication of LASIK and cataract surgery. This study was conducted among 16,471 South Korean adults aged $\geq 20$ years. Korea is a single-race country with relatively uniform genetic and environmental influences. Therefore, the results of this study are more consistent than other large population-based studies and should be broadly generalizable.

However, the following limitations must be considered. First, a cross-sectional study can identify correlation between DED and psychiatric factors, although it cannot establish a causal relationship. Second, this survey was not designed to diagnose DED, examinations related to the severity of DED were not performed. Nevertheless, many previous studies have used this data to investigate DED and its risk factors ${ }^{9,12,16}$. Third, the evaluation of sleep duration was performed through surveys. Therefore, future studies would require objective evaluation of sleep quality (actigraphy, melatonin measurement, and polysomnography) in patients with DED.

In conclusion, this study provides epidemiologic evidence that DED is associated with sleep duration, stress, and depression in the Korean population. Short sleep duration ( $\leq 5 \mathrm{~h} / \mathrm{night})$, stress perception, and depressive symptoms were significantly associated with the risk of DED even after correcting for demographic factors, lifestyle factors, and medical factors. When all three factors were present, in other words, in the case of severe mental health issues, the risk of DED increased greatly. Therefore, ophthalmologists must be aware of the possibility of stress and depression among patients with DED. 


\section{Declarations}

\section{Data Availability}

All the data supporting the conclusions of this article is included in the present article.

\section{Acknowledgments}

The authors were independent of the funders in all aspects of study design, writing of manuscript, and data analysis.

\section{Author contributions statement}

Involved in conception and design (H. K.) and conduct of the study (Y. A., H. K.); collection, management, and interpretation of data (Y. A., H. K.); data analysis (Y. A.); writing the article (Y. A., H. K.); and preparation, review, and approval of the manuscript (Y. A., H. K.). All authors reviewed the manuscript and approved the final version.

\section{Additional Information}

Competing Interests: The authors declare no competing interests

Publisher's note: Springer Nature remains neutral with regard to jurisdictional claims in published maps and institutional affiliations.

\section{References}

1. Uchino, M. \& Schaumberg, D. A. Dry eye disease: Impact on quality of life and vision. Curr. Ophthalmol. Rep, 1, 51-57 https://doi.org/10.1007/s40135-013-0009-1 (2013).

2. Lemp, M. A. \& Foulks, G. N. The definition and classification of dry eye disease. Ocul. Surf, 5, 75-92 (2007).

3. Gayton, J. L. Etiology, prevalence, and treatment of dry eye disease. Clin. Ophthalmol, 3, 405-412 https://doi.org/10.2147/opth.s5555 (2009).

4. Pflugfelder, S. C. et al. Management and therapy of dry eye disease: report of the Management and (2007). Ocul. Surf. 5, 163-178 DOI: https://doi.org/10.1016/s1542-0124(12)70085-x (2007).

5. Toda, I., Asano-Kato, N., Komai-Hori, Y. \& Tsubota, K. Dry eye after laser in-situ keratomileusis. Semin. Ophthalmol, 29, 358-362 (2014).

6. Schaumberg, D. A. et al. Patient reported differences in dry eye disease between men and women: impact, management, and patient satisfaction. PLoS One, 8, e76121 https://doi.org/10.1371/journal.pone.0076121 (2013).

7. International Dry Eye WorkShop Study Group. The epidemiology of dry eye disease: report of the epidemiology subcommittee of the international Dry Eye WorkShop (2007). Ocul. Surf. 5, 93-107 
DOI: https://doi.org/10.1016/s1542-0124(12)70082-4 (2007).

8. Patel, V. D., Watanabe, J. H., Strauss, J. A. \& Dubey, A. T. Work productivity loss in patients with dry eye disease: an online surgey. Curr. Med. Res. Opin, 27, 1041-1048 https://doi.org/10.1185/03007995.2011.566264 (2011).

9. Na, K. S., Han, K., Park, Y. G., Na, C. \& Joo, C. K. Depression, stress, quality of life, and dry eye disease in Korean women: a population-based study., 34, 733-738 https://doi.org/10.1097/IC0.0000000000000464 (2015).

10. Lu, P. et al. Dry eye syndrome in elderly Tibetans at high altitude: a population-based study in China., 27, 545-551 https://doi.org/10.1097/ICO.0b013e318165b1b7 (2008).

11. Yu, J., Asche, C. V. \& Fairchild, C. J. The economic burden of dry eye disease in the United States: a decision tree analysis., 30, 379-387 https://doi.org/10.1097/IC0.0b013e3181f7f363 (2011).

12. Ahn, J. M. et al. Prevalence of and risk factors associated with dry eye: the Korea National Health and Nutrition Examination Survey 2010-2011. Am. J. Ophthalmol, 158, 1205-1214 https://doi.org/10.1016/j.ajo.2014.08.021 (2014).

13. Kojima, T. et al. The impact of contact lens wear and visual display terminal work on ocular surface and tear functions in office workers. Am. J. Ophthalmol, 152, 933-940 https://doi.org/10.1016/j.ajo.2011.05.025 (2011).

14. Uchino, M. et al. Prevalence of dry eye disease and its risk factors in visual display terminal users: the Osaka study. Am. J. Ophthalmol, 156, 759-766 https://doi.org/10.1016/j.ajo.2013.05.040 (2013).

15. Uchino, M. et al. Prevalence and risk factors of dry eye disease in Japan: Koumi study., 118, 23612367 https://doi.org/10.1016/j.ophtha.2011.05.029 (2011).

16. Lee, W. et al. The association between sleep duration and dry eye syndrome among Korean adults. Sleep Med, 16, 1327-1331 https://doi.org/10.1016/j.sleep.2015.06.021 (2015).

17. Ayaki, M. et al. Sleep and mood disorders in women with dry eye disease. Sci. Rep, 6, 1-8 https://doi.org/10.1038/srep35276 (2016).

18. Ayaki, M. et al. Sleep disorders are a prevalent and serious comorbidity in dry eye. Invest Ophthalmol. Vis. Sci, 59, DES143-DES150 https://doi.org/10.1167/iovs.17-23467 (2018).

19. Levinson, B. A. et al. Referrals to the Wills Eye Institute Cornea Service after laser in situ keratomileusis: reasons for patient dissatisfaction. J. Cataract Refract. Surg, 34, 32-39 https://doi.org/10.1016/j.jcrs.2007.08.028 (2008).

20. Kasetsuwan, N., Satitpitakul, V., Changul, T. \& Jariyakosol, S. Incidence and pattern of dry eye after cataract surgery. PLoS One, 8, e78657 https://doi.org/10.1371/journal.pone.0078657 (2013).

21. Zheng, Y., Wu, X., Lin, X. \& Lin, H. The prevalence of depression and depressive symptoms among eye disease patients: a systematic review and meta-analysis. Sci. Rep, 7, 1-9 https://doi.org/10.1038/srep46453 (2017). 
22. Wan, K. H., Chen, L. J. \& Young, A. L. Depression and anxiety in dry eye disease: a systematic review and meta-analysis. Eye (Lond), 30, 1558-1567 https://doi.org/10.1038/eye.2016.186 (2016).

23. Ayaki, M. et al. Sleep and mood disorders in dry eye disease and allied irritating ocular diseases. Sci. Rep, 6, 1-7 https://doi.org/10.1038/srep22480 (2016).

24. Matossian, C., Song, X., Chopra, I., Sainski-Nguyen, A. \& Ogundele, A. The Prevalence and Incidence of Dry Eye Disease Among Patients Using Continuous Positive Airway Pressure or Other Nasal Mask Therapy Devices to Treat Sleep Apnea. Clin. Ophthalmol, 14, 3371 https://doi.org/10.2147/OPTH.S274949 (2020).

25. Kim, K. W. et al. Association between depression and dry eye disease in an elderly population. Invest. Ophthalmol. Vis. Sci, 52, 7954-7958 https://doi.org/10.1167/iovs.11-8050 (2011).

26. Labbé, A. et al. Dry eye disease, dry eye symptoms and depression: the Beijing Eye Study. Br. J. Ophthalmol, 97, 1399-1403 https://doi.org/10.1136/bjophthalmol-2013-303838 (2013).

27. Pouyeh, B. et al. Impact of ocular surface symptoms on quality of life in a United States veterans affairs population. Am. J. Ophthalmol, 153, 1061-1066 https://doi.org/10.1016/j.ajo.2011.11.030 (2012).

28. Kitazawa, M. The relationship of dry eye disease with depression and anxiety: a naturalistic observational study. Transl. Vis. Sci. Technol, 7, 35-35 https://doi.org/10.1167/tvst.7.6.35 (2018).

29. Galor, A. et al. Dry eye symptoms align more closely to non-ocular conditions than to tear film parameters. Br. J. Ophthalmol, 99, 1126-1129 https://doi.org/10.1136/bjophthalmol-2014-306481 (2015).

30. Kawashima, M. et al. Associations between subjective happiness and dry eye disease: a new perspective from the Osaka study. PLoS One, 10, e0123299 https://doi.org/10.1371/journal.pone.0123299 (2015).

31. Jiao, J. T. et al. Association between inflammatory cytokines and the risk of post-stroke depression, and the effect of depression on outcomes of patients with ischemic stroke in a 2-year prospective study. Exp. Ther. Med, 12, 1591-1598 https://doi.org/10.3892/etm.2016.3494 (2016).

32. Maes, M. et al. Depression and sickness behavior are Janus-faced responses to shared inflammatory pathways. BMC Med, 10, 1-19 https://doi.org/10.1186/1741-7015-10-66 (2012).

33. Mrugacz, M. et al. Pro-inflammatory cytokines associated with clinical severity of dry eye disease of patients with depression. Adv. Med. Sci, 62, 338-344 https://doi.org/10.1016/j.advms.2017.03.003 (2017).

34. Mrugacz, M. et al. Dry eye disease in patients treated with antidepressants. Klin. Oczna, 115, 111114 (2013).

35. De Salamanca, A. E. et al. Expression of muscarinic and adrenergic receptors in normal human conjunctival epithelium. Invest. Ophthalmol. Vis. Sci, 46, 504-513 https://doi.org/10.1167/iovs.040665 (2005).

36. American Academy of Sleep Medicine. International classification of sleep disorders, 3rd edition: diagnostic and coding manual (American Academy of Sleep Medicine, 2014). 
37. Thorpy, M. J. Classification of sleep disorders., 9, 687-701 (2012).

38. An, Y. \& Joo, C. K. The U-shaped association between self-reported sleep duration and visual impairment in Korean adults: a population-based study. Sleep med, 26, 30-36 https://doi.org/10.1016/j.sleep.2016.08.005 (2016).

\section{Tables}

Due to technical limitations, table 1-3 is only available as a download in the Supplemental Files section.

\section{Figures}

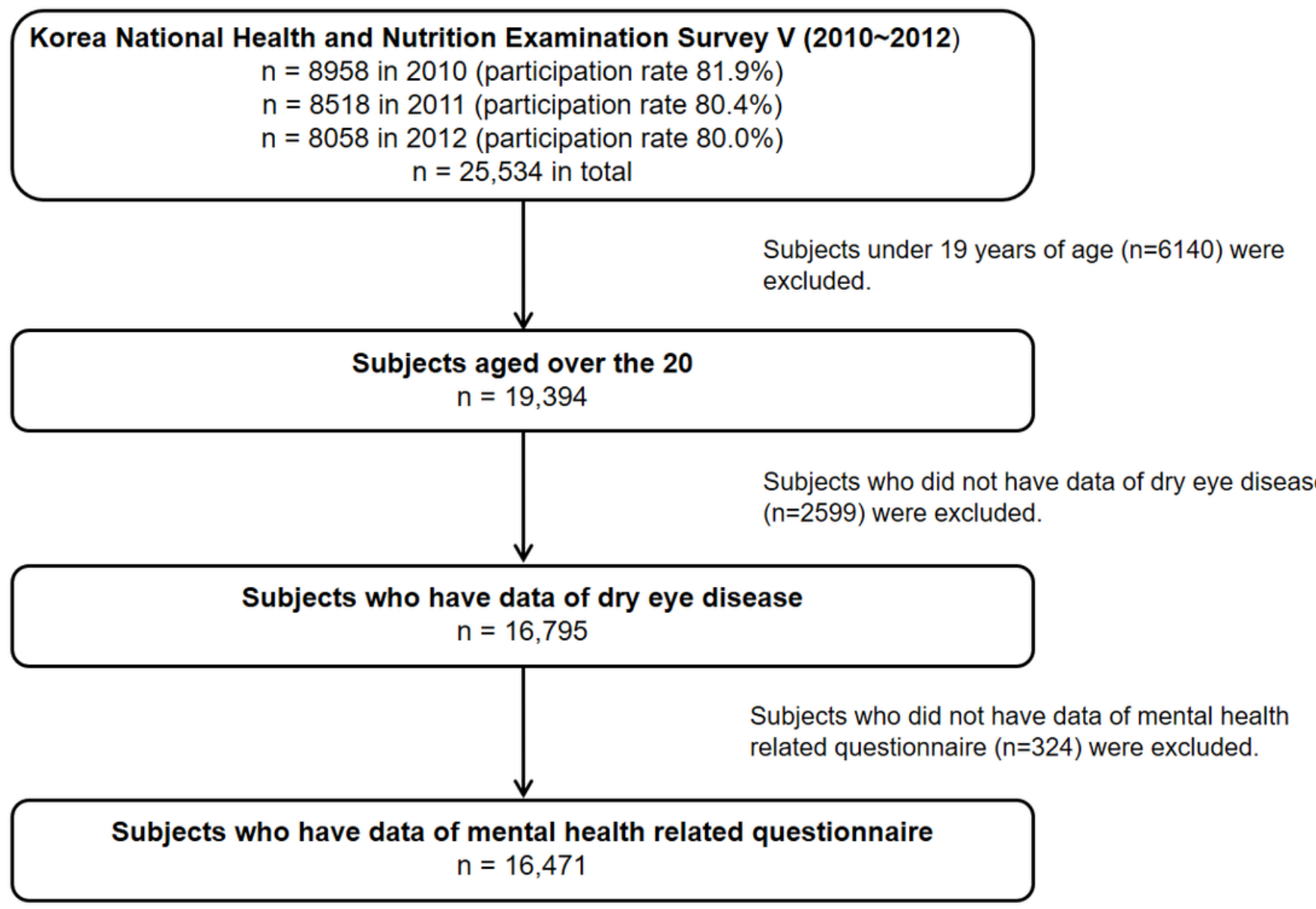

Figure 1

Flow chart of the study population. 


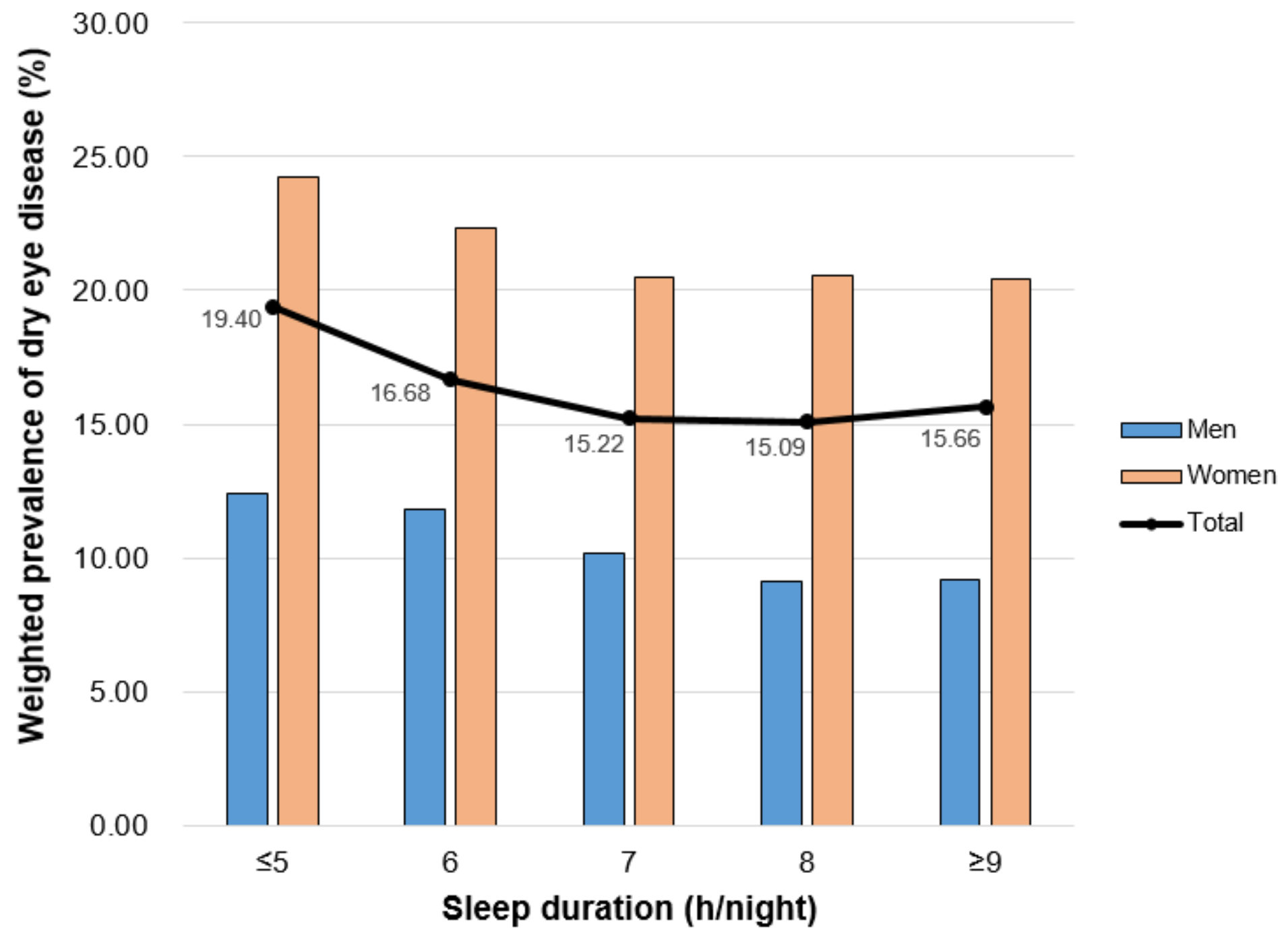

Figure 2

Relationship between sleep duration and weighted prevalence of dry eye disease in Korean adults. 
30.00

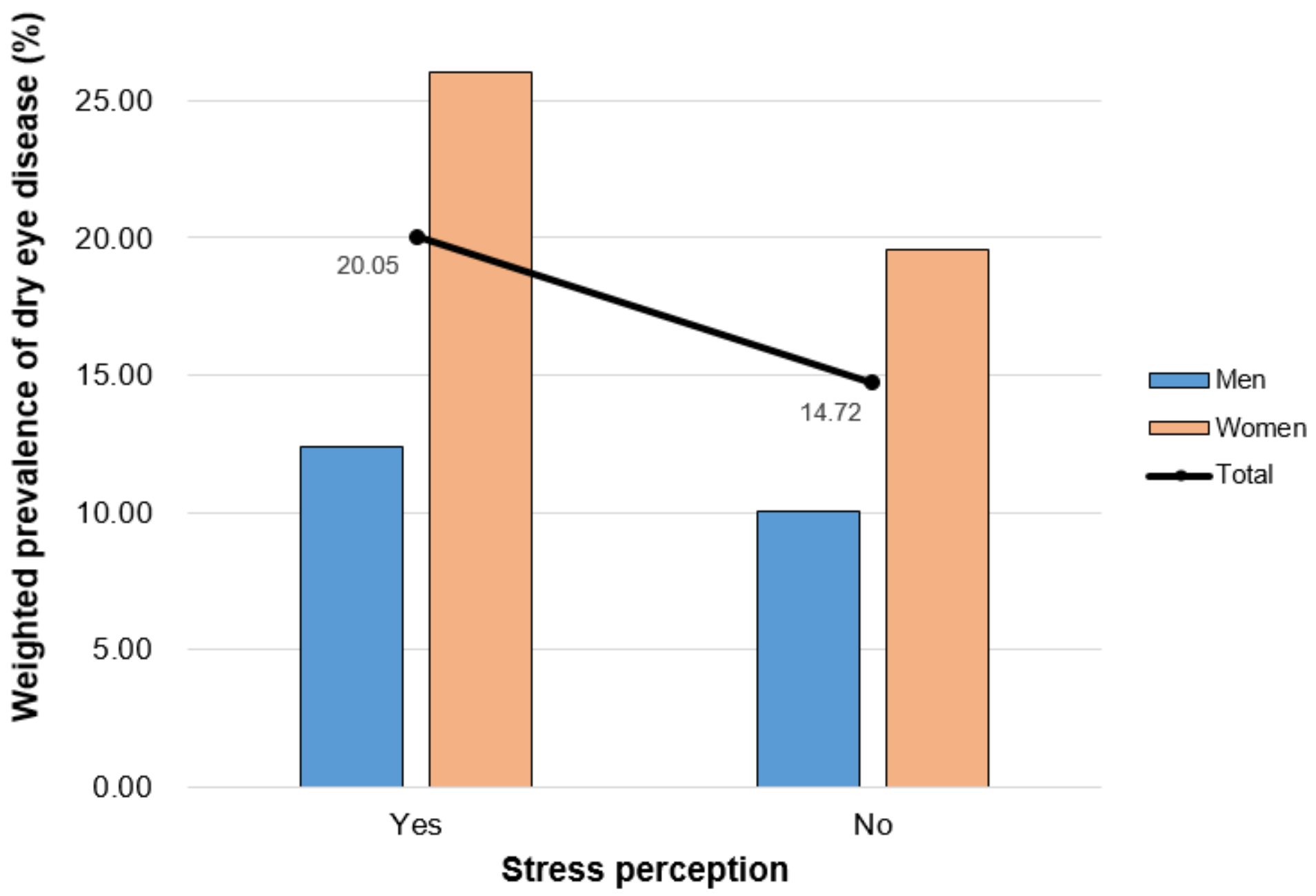

Figure 3

Relationship between stress perception and weighted prevalence of dry eye disease in Korean adults. 
30.00

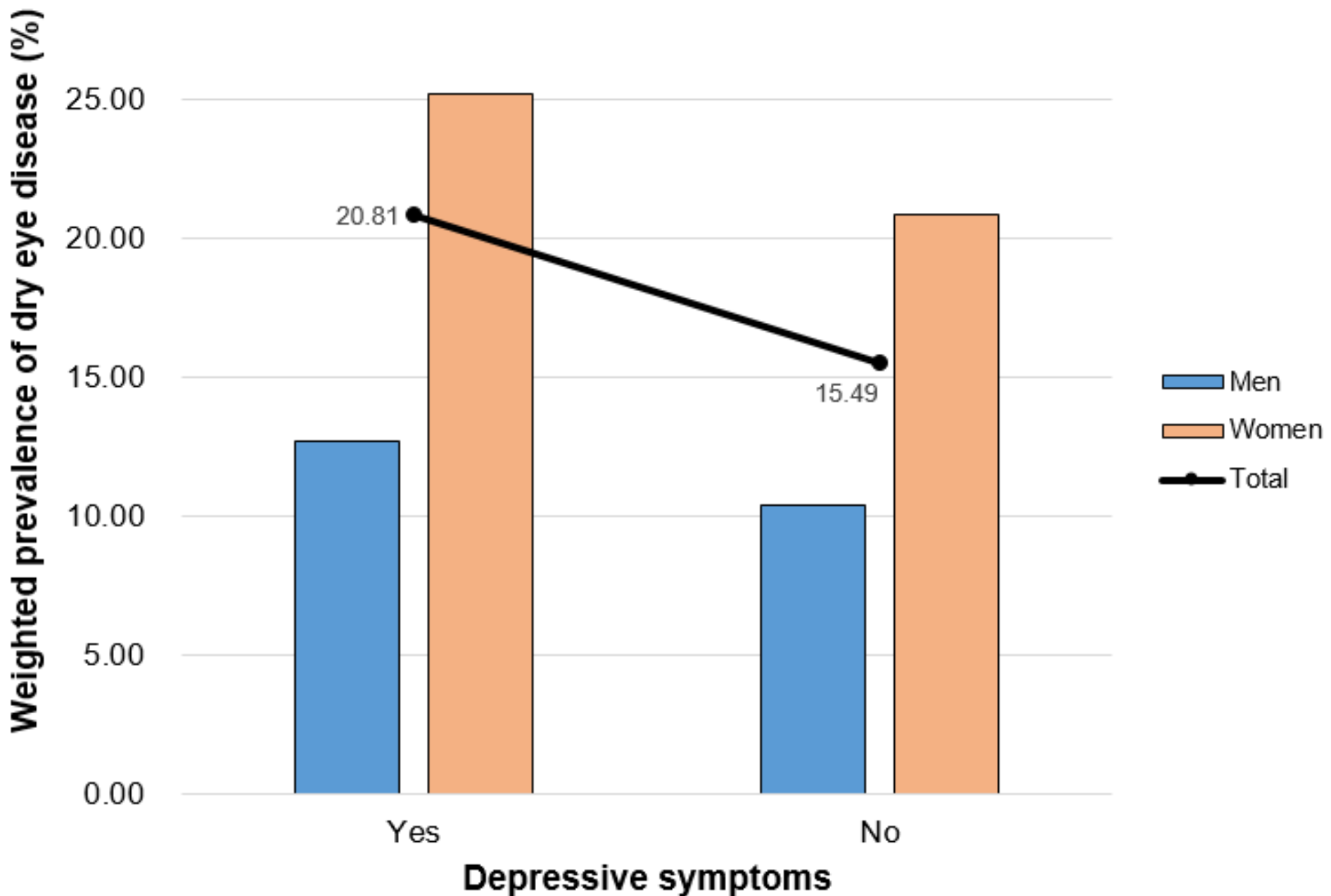

Figure 4

Relationship between depressive symptoms and weighted prevalence of dry eye disease in Korean adults. 
30.00

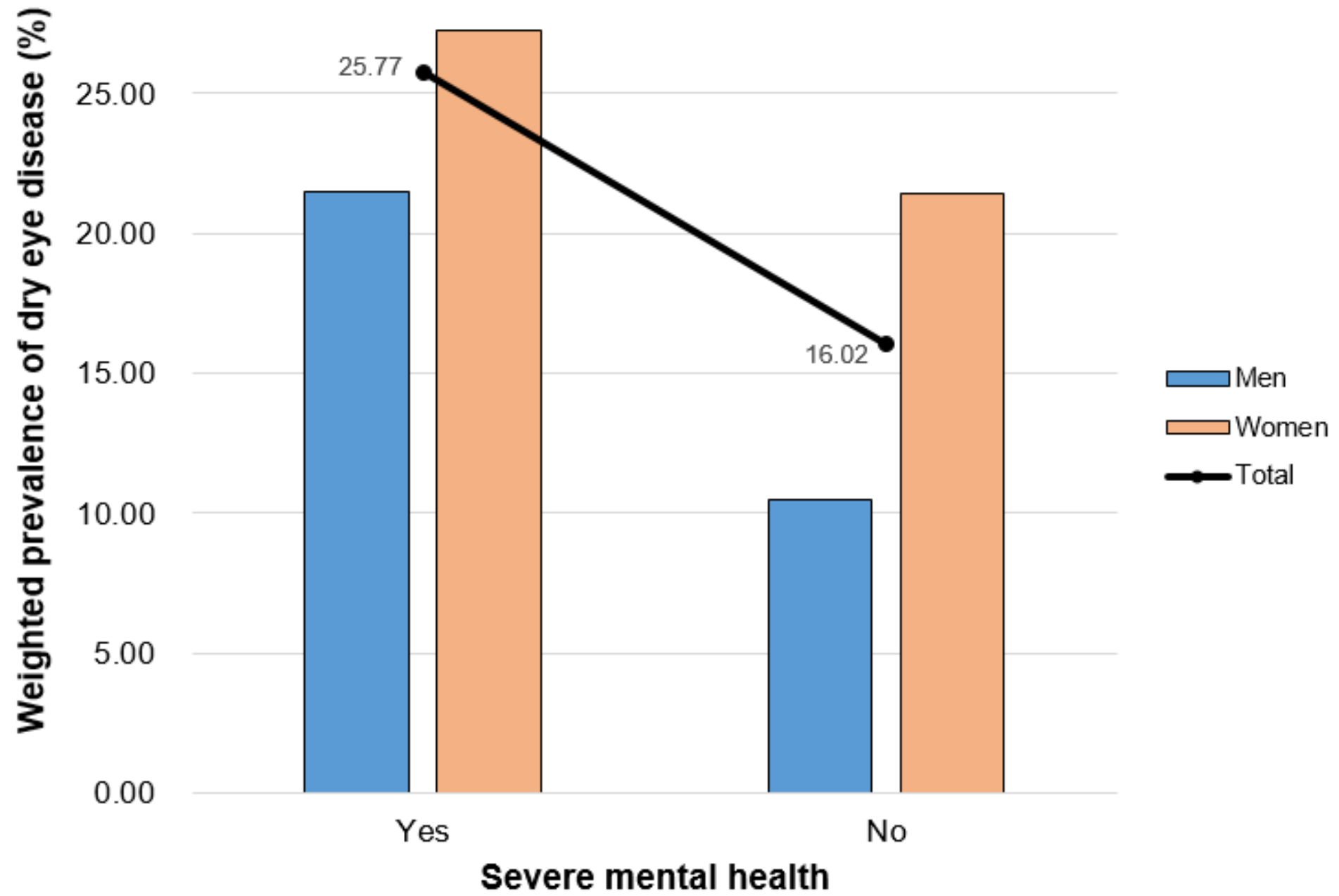

Figure 5

Relationship between severe mental health [sleep duration $(\leq 5)+$ stress perception (yes) + depressive symptoms (yes)] and weighted prevalence of dry eye disease in Korean adults.

\section{Supplementary Files}

This is a list of supplementary files associated with this preprint. Click to download.

- Tables.pdf 